\title{
Charmonium properties in the quark-gluon plasma
}

\author{
M.B. Oktay, M.J. Peardon, J.I. Skullerud ${ }^{* \dagger}$ \\ School of Mathematics, Trinity College, Dublin 2, Ireland \\ E-mail: jonivareskullerud.name

\section{G. Aarts, C.R. Allton}

Department of Physics, Swansea University, Singleton Park, Swansea SA2 8PP, Wales, UK

We present results for charmonium correlators and spectral functions in 2-flavour QCD on anisotropic lattices. Our results indicate that the S-waves $\left(J / \psi\right.$ and $\left.\eta_{c}\right)$ survive up to temperatures close to $2 T_{c}$, while the P-waves $\left(\chi_{c 0}\right.$ and $\left.\chi_{c 1}\right)$ melt away below $1.2 T_{c}$.

The XXV International Symposium on Lattice Field Theory

July 30 - August 42007

Regensburg, Germany

\footnotetext{
*Speaker.

${ }^{\dagger}$ Address after 1 Sep 2007: Department of Mathematical Physics, NUI Maynooth, Co Kildare, Ireland.
} 


\section{Introduction}

The properties of charmonium in the quark-gluon plasma is an issue of long-standing interest, following the suggestion [迎] that $J / \psi$ suppression could be a probe of deconfinement. Lattice simulations in the quenched approximation [2, 3, 5, 5] have cast doubt over this picture, indicating that $J / \psi$ may survive up to temperatures as high as $1.5-2 T_{c}$. Recently, potential model calculations using the internal energy of the heavy-quark pair have reached the same conclusion, and indicate a qualitatively similar picture in the case of $N_{f}=2$ QCD [6, 7]. This conclusion has, however, been disputed by other potential model calculations [8].

On the experimental side, data from SPS and RHIC show similar amounts of $J / \psi$ suppression at both experiments, despite the big difference in energy density. Two different scenarios have been developed to explain this result. The sequential suppression scenario [9] takes its cue from lattice results, suggesting that the entire observed suppression originates from feed-down from the excited $1 \mathrm{P}$ and $2 \mathrm{~S}$ states, which melt shortly above $T_{c}$, while the $1 \mathrm{~S}$ state survives up to energy densities higher than those reached in current experiments. On the other hand, the regeneration scenario [10, 11, 12] suggests that additional charmonium is produced at RHIC energies from coalescence of $c$ and ${ }^{-} c$ quarks originating from different pairs.

Lattice simulations with dynamical fermions ( 2 or $2+1$ light flavours) will be one of the essential ingredients in resolving several of these issues. In order to obtain a sufficient number of points in the euclidean time direction with moderate computational resources it is necessary to use anisotropic lattices, where the temporal lattice spacing $a_{\tau}$ is much smaller than the spatial lattice spacing $a_{s}$. Here we present results from $N_{f}=2$ simulations with anisotropy $\xi=a_{s} / a_{\tau}=6$.

\section{Methods}

We have carried out simulations on lattices with a temporal lattice spacing $a_{\tau} \approx(7 \mathrm{GeV})^{-1}$ and an anisotropy $\xi=a_{s} / a_{\tau}=6$, giving a spatial lattice spacing of $a_{s}=0.167 \mathrm{fm}$. Configurations have been generated at a range of temperatures, from $220 \mathrm{GeV}$ to $440 \mathrm{GeV}$, and two spatial volumes, $8^{3}(1.34 \mathrm{fm})^{3}$ and $12^{3}(2 \mathrm{fm})^{3}$. 500-1000 configurations were generated for most temperatures. The pseudocritical temperature for these lattices was estimated to be $T_{c}=205-210 \mathrm{MeV}$. For details about the anisotropic lattice action, see [13], and for further details about the high-temperature configurations, see [14].

The spectral functions $\rho_{\Gamma}(\omega)$ are related to the imaginary-time correlator $G_{\Gamma}(\tau)$ (we consider here only zero momentum) according to

$$
G_{\Gamma}(\tau)=\int_{0}^{\infty} \frac{d \omega}{2 \pi} K(\tau, \omega) \rho_{\Gamma}(\omega), \quad K(\tau, \omega)=\frac{\cosh [\omega(\tau-1 / 2 T)]}{\sinh (\omega / 2 T)} .
$$

where the subscript $\Gamma$ corresponds to the different quantum numbers. Correlators were computed in the pseudoscalar, vector, axial-vector and scalar channels, using local operators only, ie. $\Gamma=\left\{\gamma_{5}, \gamma_{i}, \gamma_{5} \gamma_{i}, 1\right\}$. Spectral functions were then determined using the maximum entropy method (MEM) [15, 16], with the modified kernel introduced in [17], which greatly improved the stability and convergence of the MEM analysis.

In addition to the spectral functions, we have also studied reconstructed correlators $G_{\mathrm{rec}}\left(\tau ; T, T_{0}\right)$, obtained by integrating (2.1) at temperature $T$ using the spectral function $\rho\left(\omega ; T_{0}\right)$ obtained at some 

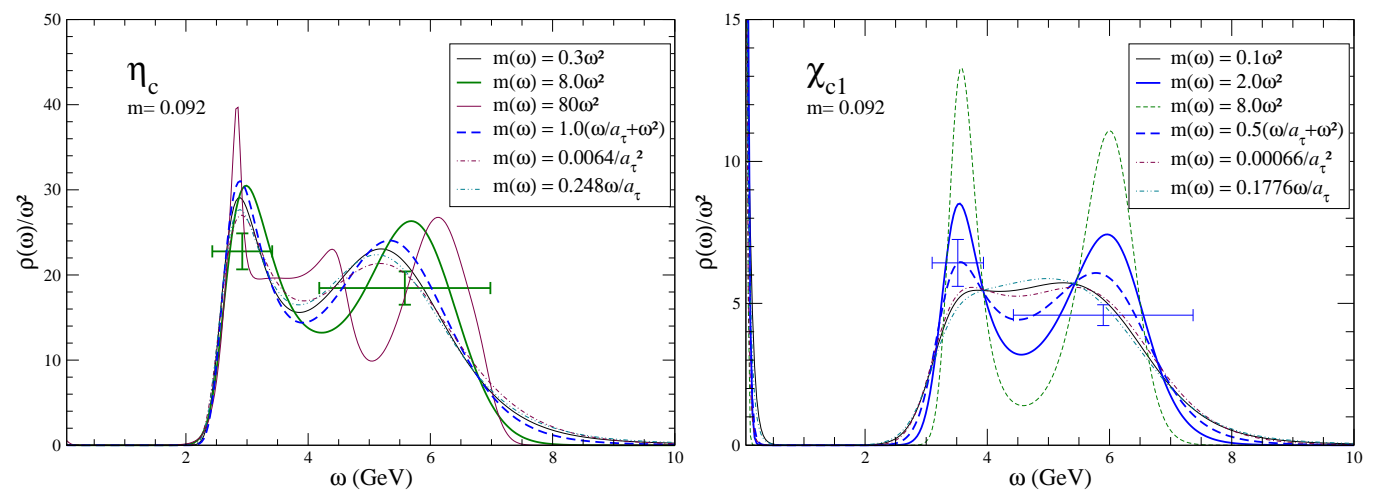

Figure 1: The pseudoscalar (left) and axial-vector (right) spectral functions for different default models, at $T=221 \mathrm{MeV}$.

reference temperature $T_{0}$. If the spectral function at $T_{0}$ can be obtained with a high degree of confidence, this may provide a more robust way of analysing possible medium modifications than by attempting to extract $\rho(\omega)$ using MEM at higher temperatures, where the number of data points decreases as $1 / T$.

\section{Results}

The pseudoscalar and axial-vector spectral functions at $T=221 \mathrm{MeV}$, just above the deconfinement transition, are shown in figure 1, for a range of different default models. In the pseudoscalar channel, we can see very little dependence on the default model: only for the default model $m(\omega)=80 \omega^{2}$ is there any significant deviation. Note that in the graph, this model would corresponds to a horizontal line at 80 , and in the absence of information from the correlators the MEM would reproduce this default model. It is natural that the tension between this rather extreme model and the data will produce some distortion in the reproduced spectral function. We conclude from this that the pseudoscalar spectral function can be reliably reproduced at this temperature. In particular, we note that the position of the first peak corresponds very closely to the zero-temperature $\eta_{c}$ mass (the charm quark mass used in this analysis was somewhat lower than the physical charm quark mass). The second peak is assumed to be a lattice artefact as its position is close to a sharp cusp in the free-field spectral function.

In the axial-vector channel, on the other hand, we find a great deal more variation. Our 'best' estimates for the spectral function (thick lines) indicate a surviving peak structure approximately at the mass of the zero-temperature $\chi_{c 1}$, but the strong default model dependence, combined with the proximity of the lattice artefact peak, makes it impossible to distinguish with any confidence between a bound state peak and a two-particle threshold.

As discussed above, we can be quite confident about our pseudoscalar spectral function at $T=221 \mathrm{MeV}$, and will therefore use this as a reference temperature for studying reconstructed correlators. The same is the case in the vector channel, while in the P-wave (scalar and axial-vector) channels the spectral functions at $T=221 \mathrm{MeV}$ are much more uncertain. We will nonetheless use this as our reference temperature also in this case. 


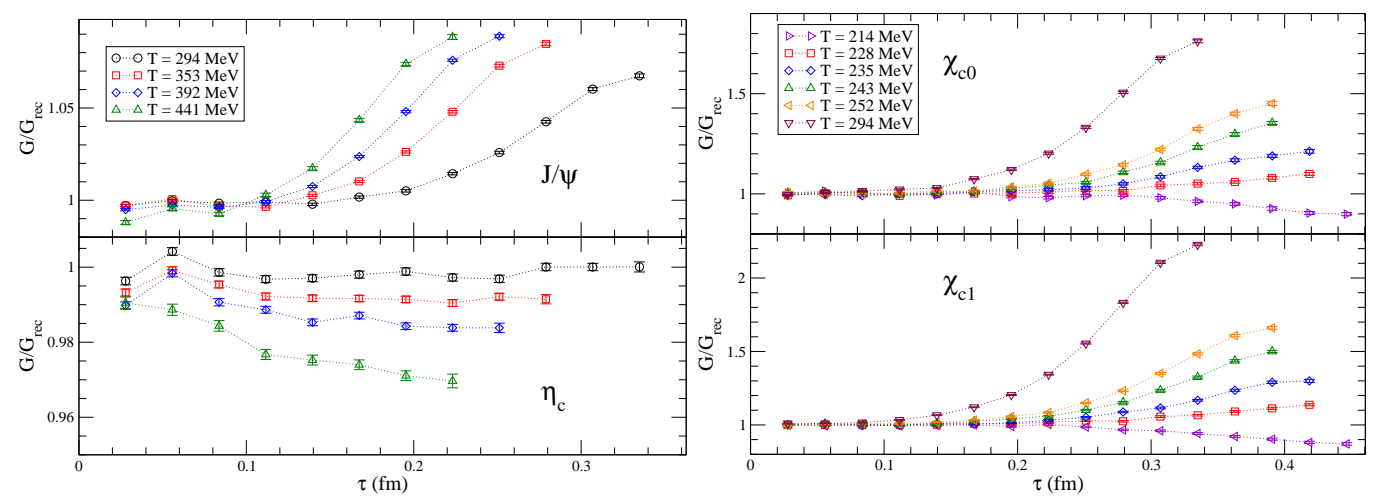

Figure 2: Reconstructed correlators for the S-wave (left) and P-wave(right) channels.
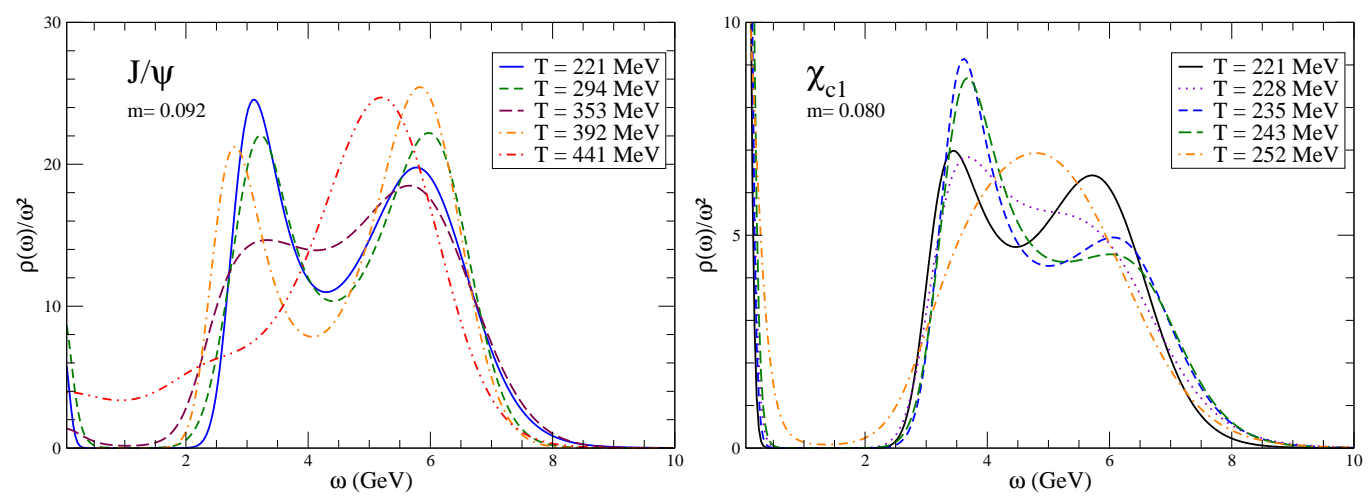

Figure 3: Spectral functions in the vector (left) and axial-vector (right) channel, for different temperatures.

The reconstructed correlators are shown in figure 2. We can see that in the pseudoscalar channel, $G_{\text {rec }}(\tau)$ changes by only $2 \%$ as the temperature is doubled with respect to $T_{0}$. In the vector channel the change is slightly larger, but still less than $10 \%$. Although it is quite possible that a null result for $G_{\text {rec }}(\tau)$ can go hand in hand with dramatic changes in the spectral functions [ 8 ], this provides prima facie evidence that medium modifications are small in these channels.

In the P-wave channels the picture is dramatically different: the long-distance correlators change by a factor 1.5-2 over a much smaller temperature range. This would indicate strong medium modifications, although a large part of the change is most likely due to the constant mode which appears in these correlators above $T_{c}[18]$. This will be investigated further in the future. It is worth noting that our results for the reconstructed correlators are very similar to those obtained in the quenched approximation for the same values of $T / T_{c}$.

Figure 3 shows spectral functions in the vector and axial-vector channel at a range of different temperatures. In the vector channel, the ground state peak appears to survive unchanged up to $T \approx 300 \mathrm{MeV}$, while at $T \approx 440 \mathrm{MeV}$ there is no sign of any surviving ground state. The default model dependence of our spectral functions becomes progressively stronger as the temperature is increased, and as a result we are not in a position at present to determine precisely where in the range $T=300-440 \mathrm{MeV}$ the $J / \psi$ melts.

The nonzero intercept at $\omega=0$ at our highest temperature could be related to a non-vanishing 
charm diffusion coefficient. We have attempted to study this using the default model $m(\omega)=$ $m_{0} \omega(b+\omega)$ which explicitly allows $\lim _{\omega \rightarrow 0} \rho(\omega) / \omega \neq 0$ [17]. However, our results at present are inconclusive, and finer lattices will in all likelihood be required to make any clear statements.

The spectral function in the pseudoscalar channel is qualitatively similar to the vector channel, but the ground state appears to survive to somewhat higher temperatures.

In the axial-vector channel there is no sign of any surviving ground state peak for $T \gtrsim 250$ $\mathrm{MeV}$ or $1.2 T_{c}$. Below this temperature there appears to be a peak structure which may correspond to a surviving $\chi_{c 1}$ state or to a threshold enhancement as discussed above. We conclude from this that the $\chi_{c 1}$ melts below $250 \mathrm{MeV}$, although a more careful analysis taking into account the constant contribution in the correlator will be required to draw any firm conclusion. A further complication is that the local operator has a poor overlap with the $\chi_{c 1}$ state, making it more difficult to extract information about the ground state if it exists. This could be improved on by using a derivative operator in the axial-vector as well as in the scalar channel. The picture in the scalar channel is similar to that in the axial-vector channel, but the uncertainty is greater.

\section{Outlook}

Our results indicate that charmonium S-waves $\left(J / \psi, \eta_{c}\right)$ survive in the quark-gluon plasma up to 1.4-2 times the critical temperature, while P-waves $\left(\chi_{c}\right)$ melt below $1.2 T_{c}$. This is conistent with what has previously been found in the quenched approximation. There are a number of uncertainties that must be addressed before firm conclusions can be drawn.

- First and foremost, the spatial lattice spacing used in this first study is fairly large $(0.17 \mathrm{fm})$. This means that the number of temporal points in the relevant temperature range is quite small, even with an anisotropy of 6 , limiting the usefulness and reliability of the maximum entropy method. Also due to the coarse lattice, lattice artefacts appear in the spectral function uncomfortably close to the physical ground state masses, obscuring the physical interpretation of the spectral functions extracted using MEM. Simulations with finer lattices are underway, which should go a long way towards addressing these issues.

- The simulation has used two degenerate flavours of sea quarks with a mass around the strange quark mass, rather than two light quarks and one strange quark as in nature. The main effects of this are to raise the (pseudo-)critical temperature above its physical value, and to inhibit charmonium dissociation by light quarks. Simulations with lighter sea quarks are planned.

- The P-waves should be probed with operators which have a better overlap with the states of interest. We are currently producing P-wave correlators using derivative operators, which we expect will provide more robust results for these channels.

We are also computing charmonium correlators at nonzero momentum. This will allow a more direct comparison with experimental results, where all $J / \psi$ particles that are observed (as dileptons) in the detectors have nonzero (transverse) momentum. Most importantly, it may inform the interpretation of the data in terms of models such as the sequential suppression model and the regeneration model, since the momentum and rapidity dependence of the $J / \psi$ yields is a key factor in distinguishing between these different models. 


\section{Acknowledgments}

This work was supported by the IITAC project, funded by the Irish Higher Education Authority under PRTLI cycle 3 of the National Development Plan and funded by IRCSET award SC/03/393Y, SFI grants 04/BRG/P0266 and 04/BRG/P0275. G.A. was supported by a PPARC advanced fellowship. We are grateful to the Trinity Centre for High-Performance Computing for their support.

\section{References}

[1] T. Matsui and H. Satz, J/ $\psi$ suppression by quark - gluon plasma formation, Phys. Lett. B178 (1986) 416.

[2] T. Umeda, K. Nomura and H. Matsufuru, Charmonium at finite temperature in quenched lattice QCD, Eur. Phys. J. C39S1 (2005) 9-26 [hep-lat/0211003].

[3] M. Asakawa and T. Hatsuda, $J / \psi$ and $\eta_{c}$ in the deconfined plasma from lattice QCD, Phys. Rev. Lett. 92 (2004) 012001 [hep-lat/0308034].

[4] S. Datta, F. Karsch, P. Petreczky and I. Wetzorke, Behavior of charmonium systems after deconfinement, Phys. Rev. D69 (2004) 094507 [hep-lat/0312037].

[5] A. Jakovác, P. Petreczky, K. Petrov and A. Velytsky, Quarkonium correlators and spectral functions at zero and finite temperature, Phys. Rev. D75 (2007) 014506 [hep-lat / 0611017 ].

[6] C.-Y. Wong, Effects of dynamical quarks on the stability of heavy quarkonia in quark-gluon plasma, hep-ph/0509088.

[7] W. M. Alberico, A. Beraudo, A. De Pace and A. Molinari, Quarkonia in the deconfined phase: Effective potentials and lattice correlators, Phys. Rev. D75 (2007) 074009 hep-ph/0612062.

[8] Á. Mócsy and P. Petreczky, Can quarkonia survive deconfinement?, arXiv:0 05.2559 [hep-ph].

[9] F. Karsch, D. Kharzeev and H. Satz, Sequential charmonium dissociation, Phys. Lett. B637 (2006) 75-80 hep-ph/0512239.

[10] P. Braun-Munzinger and J. Stachel, (Non)thermal aspects of charmonium production and a new look at J/ $\psi$ suppression, Phys. Lett. B490 (2000) 196-202 [nucl-th/0007059].

[11] R. L. Thews, M. Schroedter and J. Rafelski, Enhanced J/ $\psi$ production in deconfined quark matter, Phys. Rev. C63 (2001) 054905 hep-ph/ 0007323 .

[12] L. Grandchamp and R. Rapp, Charmonium suppression and regeneration from SPS to RHIC, Nucl. Phys. A709 (2002) 415-439 hep-ph/0205305.

[13] R. Morrin, A. Ó Cais, M. Peardon, S. M. Ryan and J.-I. Skullerud, Dynamical QCD simulations on anisotropic lattices, Phys. Rev. D74 (2006) 014505 [hep-lat/ 0604021 ].

[14] G. Aarts, C. Allton, M. B. Oktay, M. Peardon and J.-I. Skullerud, Charmonium at high temperature in two-flavor QCD, arXiv:0705.2198 [hep-lat].

[15] R. K. Bryan, Maximum entropy analysis of oversampled data problems, Eur. Biophys. J. 18 (1990) 165.

[16] M. Asakawa, T. Hatsuda and Y. Nakahara, Maximum entropy analysis of the spectral functions in lattice QCD, Prog. Part. Nucl. Phys. 46 (2001) 459-508 hep-lat/0011040]. 
[17] G. Aarts, C. Allton, J. Foley, S. Hands and S. Kim, Spectral functions at small energies and the electrical conductivity in hot, quenched lattice QCD, Phys. Rev. Lett. 99 (2007) 022002 [hep-lat/0703008].

[18] T. Umeda, A constant contribution in meson correlators at finite temperature, Phys. Rev. D75 (2007) 094502 hep-lat/0701005. 